\title{
In Situ Intestinal Perfusion of Irinotecan: Application to P-gp Mediated Drug Interaction and Introduction of an Improved HPLC Assay
}

\author{
Abdullah K. Rabba ${ }^{1}$, Luqin $\mathrm{Si}^{1}$, Kewen Xue ${ }^{1}$, Ming $\mathrm{Li}^{1}$, Gao $\mathrm{Li}^{1}$ \\ ${ }^{1}$ Department of Pharmaceutics, School of Pharmacy, Tongji Medical College, Huazhong University of Science and \\ Technology, Wuhan PR China.
}

\begin{abstract}
Purpose: To determine experimentally the intestinal permeability of the anticancer prodrug irinotecan, and to quantify the amount of its cytotoxic metabolite SN-38 that is intestinally excreted (exsorbed) as a predictor of intestinal toxicity, and to assess the effect of p-glycoprotein (p-gp) inhibitors (verapamil as a model) on the permeability and toxicity of irinotecan. Methods: Single pass intestinal perfusion of rat's whole length small intestines is applied to assess the permeability of the parent drug and quantify the intestinally excreted metabolite. The perfusion solution contained $30 \mu \mathrm{g} / \mathrm{ml}$ of irinotecan (control group) without or with verapamil (verapamil group). A simple reversed phase HPLC method with UV detection is developed and validated for simultaneous determination of irinotecan and SN-38 using camptothecin as an internal standard. Results: HPLC-UV method found to be simple, specific, accurate, and precise. Effective permeability coefficient of irinotecan found to be $4.9 \pm 1.7 \times 10^{-3} \mathrm{~mm} / \mathrm{min}$ and was doubled in verapamil group $(P=0.007)$. Average cumulative amount of SN-38 exsorbed found to be $29 \mathrm{ng} / \mathrm{cm}$ over 2 hours perfusion time which was decreased to $15 \mathrm{ng} / \mathrm{cm}$ in verapamil group $(P=0.016)$. Conculsions: in situ intestinal perfusion method was successfully applied to quantify the permeability of irinotecan and the exsorption of SN-38 in the same experiment, in a manner that robustly reflects real in vivo situation. P-gp inhibition using verapamil found to significantly enhance the intestinal permeability of irinotecan and potentially decrease the intestinal toxicity due to $\mathrm{SN}-38$ exposure.
\end{abstract}

This article is open to POST-PUBLICATION REVIEW. Registered readers (see "For Readers") may comment by clicking on ABSTRACT on the issue's contents page.

\section{INTRODUCTION}

Irinotecan (CPT-11) is a topoisomerase I inhibitor widely used for treatment of colorectal cancer. It is an ester prodrug that is biotransformed in vivo to its cytotoxic metabolite $\mathrm{SN}-38$ by the action of carboxylesterase $(1,2)$. $\mathrm{SN}-38$ is conjugated to form $\mathrm{SN}-38$-glucuronide to be excreted via bile to the intestines, where it can be hydrolyzed back to $\mathrm{SN}-38$ by the action of enterobacterial B-glucuronidase $(3,4)$; Figure 1 shows the chemical structures of irinotecan and $\mathrm{SN}-38$.

Systemic CPT-11 and SN-38 undergo excretion to the gut (exsorption) by the action of drug efflux transporters like P-glycoprotein (P-gp) (5). Both CPT-11 and SN-38 exist in $\mathrm{pH}$ dependent equilibrium between their lactone form (pharmacologically active form) in acidic media and open ring carboxylate form in alkaline media (6). Drug induced intestinal toxicity manifested as severe diarrhea is a main side effect of irinotecan found to hinder its clinical benefits (3). The intestinal toxic effect of irinotecan was shown to be associated with the intestinal exposure to the active metabolite SN-38 either by biliary excretion of SN-38 glucuronide or by intestinal excretion $(3,4)$. Drug efflux transporters mainly (p-gp) play a major role in the disposition of both CPT-11 and SN-38 (7), thus, inhibition of those intestinal efflux pumps would be a rational way to improve oral bioavailability and ameliorate intestinal toxicity of irinotecan, due to the potential of these inhibitors such as verapamil to inhibit the exsorption of both irinotecan and $\mathrm{SN}-38$ to the lumen of the gut (5). The oral route of irinotecan administration has been the subject of extensive research recently due to the promising results for overcoming the poor and erratic oral bioavailability by the action of drug efflux transport inhibitors $(8,9)$.

In situ intestinal perfusion in rats also known as single pass intestinal perfusion is a commonly used

\footnotetext{
Corresponding Author: Gao Li; E-mail address: ligaotj@163.com
} 


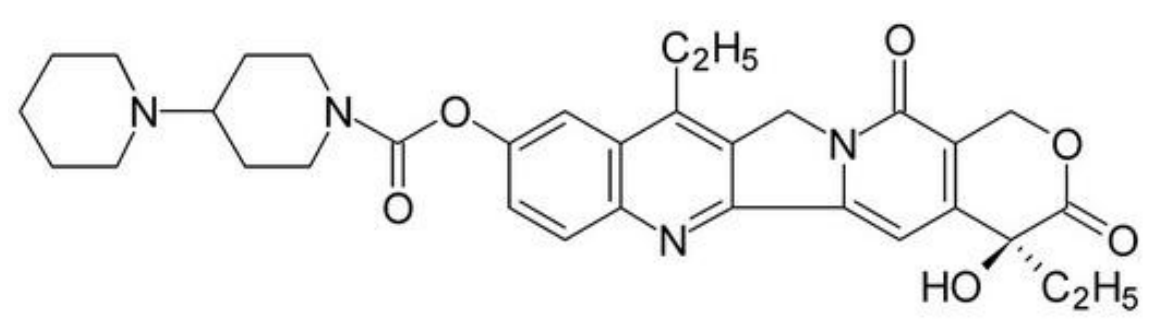

Irinotecan

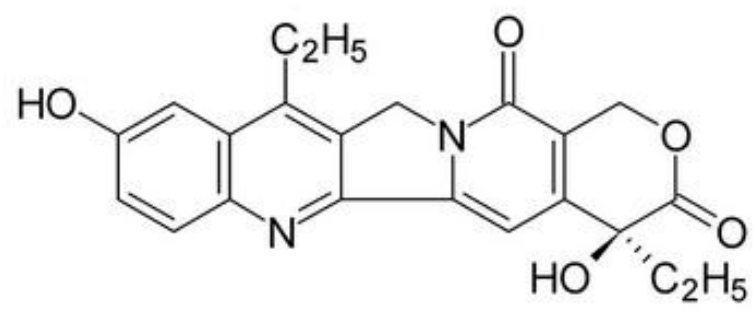

SN-38

Figure 1. Chemical structures of irinotecan (CPT-11) and its active metabolite SN-38.

technique for assessment of permeability of drugs through intestines while keeping intact blood supply to the intestinal tract throughout the experiment, making it a robust tool for simulating real in vivo conditions following oral drug administration (10-13). The improved form of this technique, where the whole small intestines is used for more accurate determination of drug's permeability, especially for low permeability compounds, has been recently introduced in our lab and published elsewhere (14).

HPLC methods for determination of both CPT-11 and SN-38 in biological fluids have already been described. Many of them required complicated and expensive equipments such as LC-MS or fluorescence detectors with the capability of switching wavelength (15-17]). UV detection is considered to be simple and most accessible for the research community. A recently reported gradient elution HPLC-UV analysis method for CPT-11 and SN-38 required a relatively long run time of $35 \mathrm{~min}$ (18).

In this report we are designing an in situ experiment for assessing both permeability and intestinal toxicity of irinotecan utilizing the improved in situ intestinal perfusion model and applying this model to study the potential effect of efflux transport inhibitors on the absorption and intestinal toxicity of irinotecan. To achieve this goal we developed and validated a new, simple, and rapid isocratic reversed phase HPLC-UV method for simultaneous determination of CPT-11 and SN-38 in the lumen of rat's small intestines following in situ intestinal perfusion of irinotecan. And to assess the effect of efflux transporter inhibition on the permeability and toxicity of irinotecan the widely used $\mathrm{p}$-gp inhibitor verapamil was used as a model p-gp inhibitor.

To our best knowledge there is no available published work that uses the in situ perfusion model to study the permeability and intestinal toxicity of irinotecan.

\section{METHODS}

\section{Materials}

Irinotecan hydrochloride was a gift from Jiang $\mathrm{Su}$ Heng Rui Medicine Co. Ltd. (China). SN-38 and camptothecin were purchased from Cheng $\mathrm{Du}$ Lanbei Phytochemistry Science and Technology Co. Ltd. (china).Verapamil hydrochloride was obtained from Centralpharm Inc. (china). DMSO and acetonitrile obtained from Sinopharm Chemical Reagent Co. Ltd. (china). All other chemicals and reagents were of HPLC or analytical reagent grade. 
Instruments and Chromatographic Conditions For chromatographic analysis a Hitachi HPLC System (Kyoto, Japan) was used, which was equipped with a quaternary gradient flow pressure mixing pump (L-2130), and a UV detector (L-2400). A Knauer Eurospher 100-5 C 18 chromatographic column $(250 \mathrm{~mm} \times 4.0 \mathrm{~mm})$ was used for separation, with a $\mathrm{C}_{18}(4 \mathrm{~mm} \times 10 \mathrm{~mm}$ i.d., $10 \mu \mathrm{m})$ pre-column from Knauer (Berlin, Germany).

The mobile phase was acetonitrile: $20 \mathrm{mM}$ $\mathrm{KH}_{2} \mathrm{PO}_{4}(35: 65, \mathrm{v}: \mathrm{v})$ with final $\mathrm{pH}$ adjusted to 3.5 using phosphoric acid. Prior to use, the mobile phase was filtered through a $0.22 \mu \mathrm{m}$ microporous membrane (phenomenon technology development Co. Ltd. (china)), and degassed by ultrasonication.

Separations were performed at room temperature $\left(25 \pm 2{ }^{\circ} \mathrm{C}\right)$ and a flow rate of $1.0 \mathrm{~mL} / \mathrm{min}, 50 \mu \mathrm{L}$ was injected into the system for analysis. The wave length of UV detection was set at $380 \mathrm{~nm}$. The structurally related compound camptothicin (CPT) was used as an internal standard (IS).

\section{Preparation of Stock and Standard Solutions}

Individual stock solutions of CPT-11, SN38 and CPT $(2 \mathrm{mg} / \mathrm{mL})$ were prepared in DMSO, divided into aliquots and stored at $-20^{\circ} \mathrm{C}$. standard calibration solutions and quality control samples were prepared daily by diluting aliquots from the stock solution into blank perfusion solution (perfused through rat's small intestines). Internal standard (IS) solution was a solution of CPT 10 $\mu \mathrm{g} / \mathrm{ml}$ in $6 \%$ perchloric acid.

\section{Sample Preparation}

Frozen intestinal perfusion samples were thawed at room temperature and $200 \mu \mathrm{L}$ of IS solution were added to $200 \mu \mathrm{L}$ of the perfusion sample and vortexed for $1 \mathrm{~min}$ then centrifuged at $12000 \mathrm{rpm}$ for $10 \mathrm{~min}$. The addition of IS solution that contain $6 \%$ perchloric acid is enough for the conversion of the carboxylate forms of CPT-11 and SN-38 to their lactone forms, and to precipitate protein impurities that could be present as a result of the intestinal perfusion process.

\section{Animals}

Male Sprague-Dawley rats (220-280 g) were obtained from the Experimental Animal Supply Center of Tongji Medical College, Huazhong University of Science and Technology, Wuhan, China. Rats were acclimatized for one week under controlled conditions including a light/dark cycle of 12 hours, and a temperature of $25^{\circ} \mathrm{C}$. Food and water were provided on an ad libitum basis. All animal experiments were performed in strict accordance with the Guidelines for Animal Experimentation of Tongji Medical College, Huazhong University of Science and Technology, Wuhan, China.

\section{Composition of the Perfusion Solution}

The perfusion solution consisted of $10 \mathrm{mM}$ D-glucose, $120 \mathrm{mM} \mathrm{NaCl}, 4.6 \mathrm{mM} \mathrm{KCl}, 1.5 \mathrm{mM}$ $\mathrm{NaH}_{2} \mathrm{PO}_{4}, 0.7 \mathrm{mM} \mathrm{Na} \mathrm{HPO}_{4}, 0.5 \mathrm{mM} \mathrm{MgCl}$, and $15 \mathrm{mM} \mathrm{NaHCO}_{3}$ Per Liter of distilled water. Aliquots from the stock solution of irinotecan were added to achieve a CPT-11 concentration of $30 \mu \mathrm{g} / \mathrm{ml}$ and $1.5 \% \mathrm{v} / \mathrm{v}$ of DMSO, (Venkatesh G et al) demonstrated that DMSO levels of up to $5 \%$ did not interfere with the permeability parameters obtained from SPIP experiments (19). For the P-gp inhibition experiments $150 \mu \mathrm{g} / \mathrm{ml}$ of verapamil hydrochloride was used. The $\mathrm{pH}$ of the perfusion solution was adjusted to 6.5 using phosphoric acid. Preliminary experiments showed that no considerable adsorption of the compounds on the tubing and other apparatus took place.

\section{Improved In Situ Single Pass Intestinal Permeability}

The improved in situ perfusion studies were performed using established method described previously (14), with minor modifications. Prior to the surgical procedure, the rats were fasted overnight $(16-20 \mathrm{~h})$ with water ad libitum. The rats were anesthetized with intraperitonial injection of urethane $(150 \mathrm{mg} / 100 \mathrm{~g}$ body weight $)$ and placed on a heating pad to maintain normal body temperature $\left(37^{\circ} \mathrm{C}\right)$. The intestines of the rats were exposed by a midline abdominal incision. Bile duct was ligated to exclude the effect of biliary excretion. During surgical procedure great deal of care was taken so as not to interrupt blood circulation of the intestinal tract. Proximal and distal ends of the small intestines were identified and canulated using silicone tubing ( $4 \mathrm{~mm}$ diameter) for inlet and outlet perfusion respectively. Small intestines were covered with a wet pad throughout the experiment to prevent dehydration. Normal saline solution $\left(37^{\circ} \mathrm{C}\right)$ was gently pumped through the inlet tubing, to rinse the intestines, until the outlet is clear. Inlet tubing was attached to peristaltic pump (Petro Gas Ausrüstungen, Berlin, 
Germany), with Perfusion solution $\left(37^{\circ} \mathrm{C}\right)$ continuously pumped at a flow rate of $0.3 \mathrm{ml} / \mathrm{min}$ for $180 \mathrm{~min}$. Collection of perfusate was done following a pre established schedule i.e. 10 minutes intervals beginning after 60 minutes of continuous perfusion. Achievement of steady state levels of irinotecan in the perfusate from pilot experiments found to be 60-90 minutes. At the end of the experiments the animal was euthanized by a single lethal intracardiac dose of anesthetic (urethane) and the length of the intestinal segment was measured. Net water flux (NWF) in the rat in situ experiment was quantified by weight and volume measurements $(13,14)$. Samples were kept frozen at $-20^{\circ} \mathrm{C}$ until analyzed.

\section{DATA ANALYSIS AND STATISTICS}

Effective permeability coefficient $\left(P_{\text {eff }}\right)$ of Irinotecan was calculated from the steady-state concentrations of the compound in the perfusate collected after correction for NWF. Permeability values were calculated using the following equation according to the parallel tube model (20):

$$
\mathrm{P}_{\mathrm{eff}}=\frac{-Q \ln \left[C_{\text {out }(\text { corrected })} / C_{\text {in }}\right]}{2 \pi r l}
$$

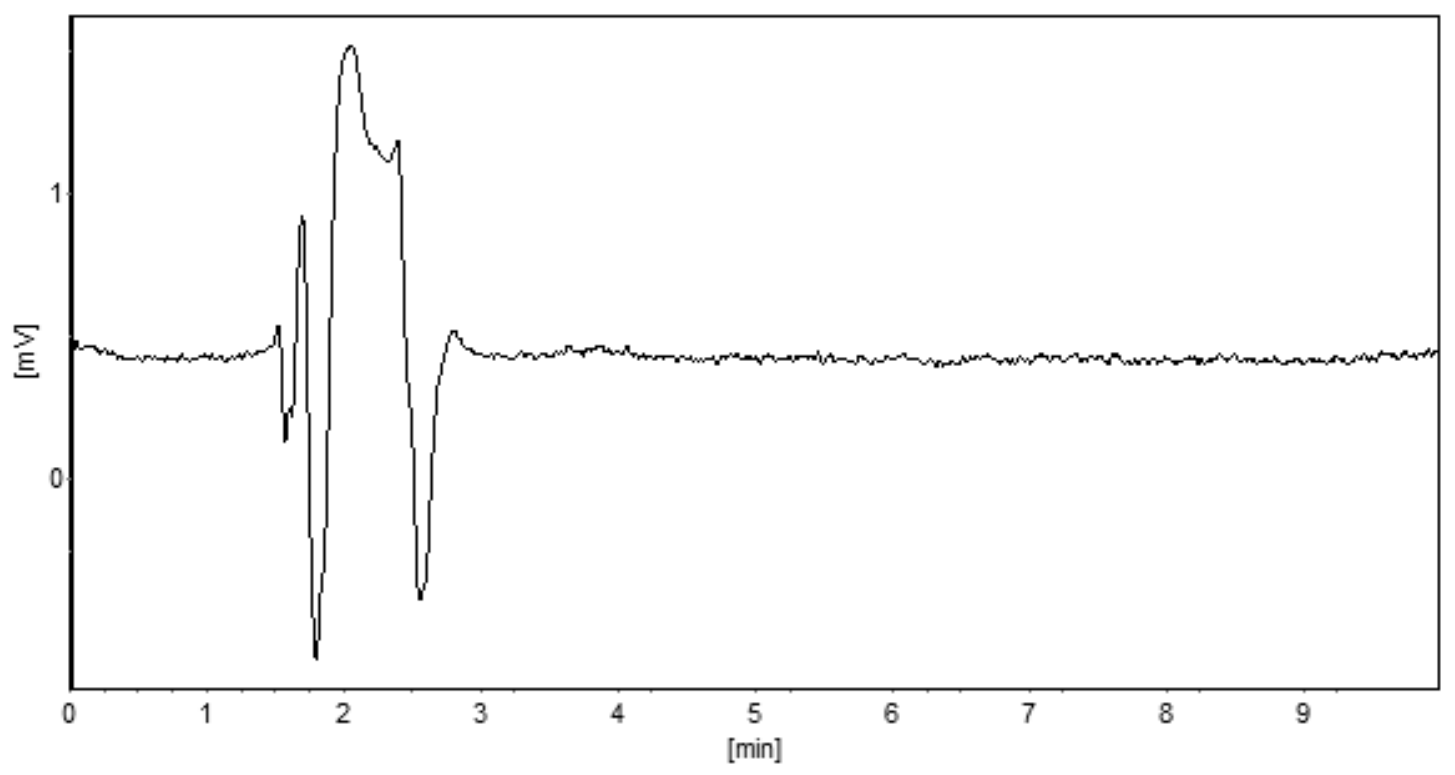

Where $C_{\text {in }}$ is the inlet concentration and $C_{\text {out (corrected) }}$ is the outlet concentration of compound, which is corrected for volume change in intestines using weight and density measurements. $Q$ is the flow rate $(0.3 \mathrm{ml} / \mathrm{min}), r$ is the rat intestinal radius $(0.18$ $\mathrm{cm})(20)$, and $l$ is the length of the intestines measured after completion of the experiment. The exsorbed SN-38 measured in the outlet perfusate was used as a potential predictor of intestinal toxicity of irinotecan. All values were presented as mean \pm standard deviation of 6 different animals. For comparing the two groups for statistical significance, one way ANOVA was used. $P$ values less than 0.05 considered as statistically significant.

\section{HPLC Method validation}

\section{Specificity}

Specificity evaluation was carried out using five different blank rat perfusion samples that were treated the same way real experiment samples being treated, and injected into the HPLC to assess whether or not endogenous constituents might interfere at the retention time of both analytes (CPT-11 and SN-38) and internal standard (CPT). Figure 2 shows a representative chromatogram of a blank perfusion sample.

Figure 2 a representative chromatogram of a blank perfusion solution that was perfused through the rat's small intestines revealing the absence of interfering peaks. 


\section{Linearity}

Seven-point linearity curves were constructed for three consecutive days for each analyte. A correlation of more than 0.999 was obtained for both linearity curves.

\section{Accuracy and precision}

The accuracy of the method was established by using quality control samples at low, medium and high concentrations of $2.5,25$ and $50 \mu \mathrm{g} / \mathrm{mL}$ for CPT- 11 and $0.05,0.5$ and $2 \mu \mathrm{g} / \mathrm{mL}$ for SN-38. All the samples were prepared in five replicates. Accuracy was calculated using the formula $\% \mathrm{RE}=$ $(E-\mathrm{T})(100 / T)$ where $E$ is the experimentally determined concentration and $T$ is the theoretical concentration. Precision was assessed by calculating inter and intra-day variability of quality control samples at low, medium and high concentrations. Inter-day data was obtained by analyzing the quality control samples on three consecutive days of assay, while the intra-day precision data was obtained by analyzing three sets of quality control samples in a single day. Assay precision was calculated using the formula \%RSD $=(\mathrm{SD} / M) \times 100$ where $M$ is the mean of the experimentally determined concentrations and SD is the standard deviation of $M$.

\section{Recovery}

The percentage recovery was determined by using quality control samples at low, medium and high concentrations of $2.5,25$ and $50 \mu \mathrm{g} / \mathrm{mL}$ for CPT- 11 and $0.05,0.5$ and $2 \mu \mathrm{g} / \mathrm{mL}$ for SN-38. Recovery was calculated by comparing the mean ratio of (peak area of analyte)/(peak area of internal standard) of five replicates $(n=5)$ of spiked blank perfusion samples to those spiked in mobile phase.

\section{Stability studies}

Stability experiments were performed with low, medium and high quality control samples to evaluate the stability of both analytes in blank perfusion samples under different conditions by simulating the same conditions that encountered during our experiment's sample analysis. Experiments were performed to determine on-bench $\left(25 \pm 2^{\circ} \mathrm{C}\right)$ stability (after $8 \mathrm{~h}$ ), three (freeze-thaw) cycles stability and short-term stability at $-20^{\circ} \mathrm{C}$ for 30 days.

\section{Lower limits of detection and quantification}

Lower limit of detection (LOD) and lower limit of quantification (LOQ) where approached based on the standard deviation of the response and the slope of the calibration curve according to the following formulae (ICH guidelines) (21):

$$
\begin{aligned}
& L O D=\frac{3.3 \sigma}{S} \\
& L O Q=\frac{10 \sigma}{S}
\end{aligned}
$$

Where $\sigma=$ the standard deviation of the response which was estimated as the standard deviation of $\mathrm{y}$-intercepts of 3 calibration regression lines, and $\mathrm{S}$ $=$ the slope of the calibration curve

\section{RESULTS}

\section{Chromatographic Method}

Following a brief sample treatment step that resulted in protein precipitation as well as conversion of the open ring carboxylate form of the two analytes to their closed ring lactone form, good separations were achieved within $10 \mathrm{~min}$. validation parameters all found to be within the accepted range as explained in the following subsections.

\section{Specificity}

The optimum single UV wavelength for detection of the two analytes (CPT-11 and SN-38) and the internal standard (CPT) simultaneously with adequate sensitivity was found to be $380 \mathrm{~nm}$, since a fixed wavelength detector was used. There were no interfering peaks of endogenous compounds observed at the retention times of the two analytes and the internal standard in blank perfusion sample. Retention times found to be 3.7, 6.6, and 9.0 minutes for CPT-11, SN-38, and CPT, respectively. A representative chromatogram is shown in Figure 3.

\section{Linearity}

Samples were quantified using the concentration response relationship given by the simple regression analysis: $y=m x+c$, with the response being the peak area of the analyte to that of the internal standard. The correlation coefficients of the calibration curves for both analytes were more than 0.999. Linearity parameters for CPT-11 and SN-38 are shown in Table 1. 


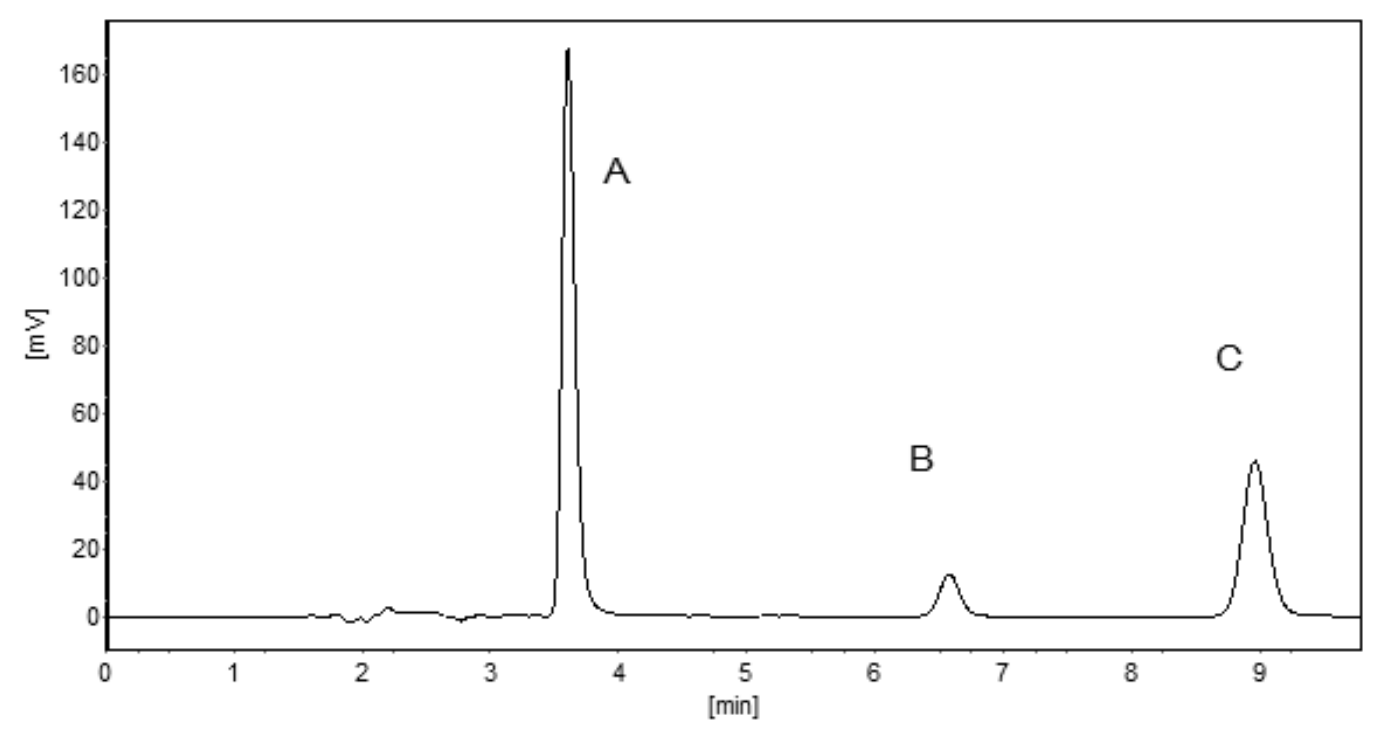

Figure 3. Representative chromatogram of a sample from intestinal perfusion containing (A: 31.25ug/ml CPT-11, B: $1.25 \mathrm{ug} / \mathrm{ml} \mathrm{SN-38,} \mathrm{C:} \mathrm{Internal} \mathrm{Standard} \mathrm{camptothecin.}$

Table 1. Linearity parameters of CPT-11 and SN-38 expressed as mean (standard deviation) of three calibration curves

\begin{tabular}{lllll}
\hline \multicolumn{1}{c}{ Analyte } & \multicolumn{1}{c}{ Range $(\mu \mathrm{g} / \mathrm{mL})$} & \multicolumn{1}{c}{ Slope } & \multicolumn{1}{c}{ Intercept } & \multicolumn{1}{c}{ Correlation coefficient } \\
\hline CPT-11 & $(0.98-62.5)$ & $0.0626(0.0015)$ & $0.01147(0.0077)$ & $0.9997(0.0002)$ \\
SN-38 & $(0.04-2.5)$ & $0.1786(0.0139)$ & $0.0005(0.0001)$ & $0.9996(0.0002)$ \\
\hline
\end{tabular}

\section{Accuracy and precision}

For CPT-11 and SN-38 the intra-day accuracy was $\leq 7.49 \%$ and $\leq 7.36 \%$, respectively, while the intra-day precession was $\leq 2.71 \%$ for CPT- 11 and $\leq 2.98 \%$ for SN-38. The inter-day accuracy was $\leq 7.21 \%$ and $\leq 6.14 \%$ for CPT-11 and SN-38, respectively, while the inter-day precession was $\leq 1.95 \%$ and $\leq 3.19 \%$ for CPT-11 and SN-38 respectively. The accuracy and precision results for both intra- and inter-day are shown in Table 2.

Table 2. Intra and inter-day precision expressed as \%RSD and accuracy expressed as \%RE obtained from three calibration curves with three levels of quality control samples of CPT-11 and SN-38.

\begin{tabular}{|c|c|c|c|c|c|c|}
\hline \multicolumn{5}{|c|}{ Intra-day (5 replicates) } & \multicolumn{2}{|c|}{ Inter-day(3 days) } \\
\hline $\begin{array}{c}\text { CPT-11 spiked } \\
\text { concentration }(\mu \mathrm{g} / \mathrm{mL})\end{array}$ & $\begin{array}{c}\text { Mean measured } \\
\text { concentration }(\mu \mathrm{g} / \mathrm{mL})\end{array}$ & $\begin{array}{c}\text { Standard } \\
\text { deviation }(\mu \mathrm{g} / \mathrm{mL})\end{array}$ & $\% \mathrm{RE}$ & $\% \mathrm{RSD}$ & $\% \mathrm{RE}$ & $\% \mathrm{RSD}$ \\
\hline 2.5 & 2.69 & 0.073 & 7.49 & 2.71 & 7.21 & 1.21 \\
\hline 25 & 25.42 & 0.606 & 1.66 & 2.39 & 3.44 & 1.64 \\
\hline 50 & 50.85 & 0.547 & 1.71 & 1.07 & 3.00 & 1.95 \\
\hline \multicolumn{7}{|c|}{$\begin{array}{c}\text { SN-38 spiked } \\
\text { conccentration }(\mu \mathrm{g} / \mathrm{mL})\end{array}$} \\
\hline 0.05 & 0.0537 & 0.0016 & 7.36 & 2.98 & 6.14 & 3.19 \\
\hline 0.50 & 0.5130 & 0.0065 & 2.61 & 1.27 & 4.43 & 3.01 \\
\hline 2.00 & 2.0766 & 0.0197 & 3.83 & 0.94 & 4.23 & 1.99 \\
\hline
\end{tabular}




\section{Recovery}

The percentage recovery for CPT-11 was $106.2 \%$, $100.8 \%$, and $101.2 \%$, for low, medium, and high quality control concentrations, respectively. For SN-38 the recovery of low, medium, and high concentrations, was $100.4 \%, 102.9 \%$, and $99.7 \%$, respectively.

\section{Stability}

Stability assessment for CPT-11 and SN-38 was carried at low, medium and high quality control concentrations. Both analytes were found to be stable in the blank perfusion sample for $8 \mathrm{~h}$ at room temperature $\left(25 \pm 2^{\circ} \mathrm{C}\right)$ and after three cycles of freezing (at $-20^{\circ} \mathrm{C}$ ) and thawing (at room temperature). The samples were stored at $-20^{\circ} \mathrm{C}$ for short-term stability studies and found to be stable for at least 30 days. Stability results are summarized in Table 3.

\section{Lower limits of detection and quantification}

LOD and LOQ for SN-38 calculated to be 0.0018 and $0.0056 \mu \mathrm{g} / \mathrm{ml}$ respectively. Corresponding concentrations were spiked and analyzed practically to assess the validity of the calculation method, and found to be within the accepted range of accuracy. For application to our in situ experiment there was no need to determine concentrations of CPT-11 lower than those used in the linearity and accuracy assessment, so, LOQ for CPT-11 set to be the lowest concentration used in this method validation's linearity assessment i.e. $0.98 \mu \mathrm{g} / \mathrm{ml}$.

\section{Rat's Single Pass Intestinal Perfusion}

Applying the above mentioned validated HPLC method we successfully determined the level of the parent drug irinotecan and its active metabolite SN-38 following rats intestinal perfusion of irinotecan (control group) or irinotecan plus the p-glycoprotein inhibitor verapamil (verapamil group). The concentration of CPT-11 in the perfusate was used to calculate the effective permeability coefficient $\mathrm{P}_{\text {eff }}$ of irinotecan which found to be $4.9 \pm 1.7 \times 10^{-3} \mathrm{~mm} / \mathrm{min}$ that corresponded to an outlet:inlet drug concentration of $0.88 \pm 0.03$. When verapamil was coperfused with irinotecan $\mathrm{P}_{\text {eff }}$ increased to $10.4 \pm 3.4 \times 10^{-3}$ $\mathrm{mm} / \mathrm{min}(P=0.0073)$, Figure 4 , and the outlet:inlet drug concentration decreased to $0.72 \pm 0.08$ $(P=0.0014)$.

In control group the average cumulative amount of SN-38 exsorbed found to be $29 \mathrm{ng} / \mathrm{cm}$ over 2 hours perfusion time which was decreased to $15 \mathrm{ng} / \mathrm{cm}$ in verapamil group $(P=0.016)$, Figure 5 shows the cumulative exsorbed $\mathrm{SN}-38$ in the two groups.

\section{DISCUSSION}

Compared to the previously described analytical methods for determination of CPT-11 and SN-38, this isocratic HPLC-UV method found to be advantageous in terms of simplicity and feasibility. HPLC-UV instruments are considered to be, for most researchers, more accessible and feasible than LC-MS, HPLC with switching wavelength fluorescence detectors, or even HPLC with conventional fluorescence detectors, which is the case in our lab.

\begin{tabular}{cccc}
\hline \multicolumn{2}{c}{ Table 3. Stability data of quality control samples of CPT-11 and SN-38 at different conditions expressed as \%RE. } \\
\hline $\begin{array}{c}\text { CPT-11 spiked concentration } \\
(\mu \mathrm{g} / \mathrm{mL})\end{array}$ & $\begin{array}{c}\text { Benchtop stability } \\
\text { freeze-thaw } \\
\text { cycles }\end{array}$ & $-20^{\circ} \mathrm{C}$ for 30 days \\
2.5 & -3.22 & -5.18 & -5.77 \\
25 & -2.42 & -3.44 & -3.74 \\
50 & 1.53 & -1.86 & -3.91 \\
$\mathrm{SN}-38$ spiked conccentration $(\mu \mathrm{g} / \mathrm{mL})$ & & & -4.22 \\
0.05 & -4.69 & -5.61 & -2.73 \\
0.50 & -1.17 & -2.48 & 3.43 \\
\hline
\end{tabular}




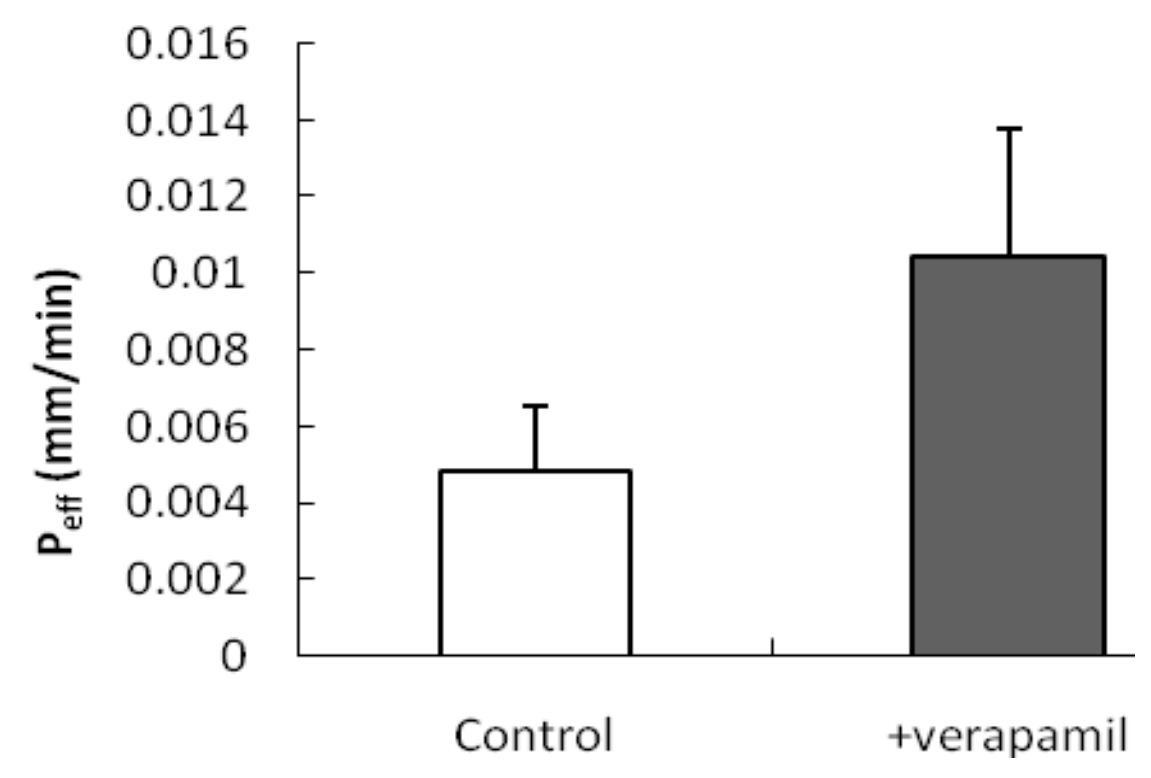

Figure 4. Effective permeability coefficient $\left(\mathrm{P}_{\mathrm{eff}}\right)$ of irinotecan obtained from in situ perfusion experiments in rats with (+verapamil) and without (Control) the P-gp inhibitor verapamil $(P=0.0073)$, error bars represent the standard deviation of 6 readings each.

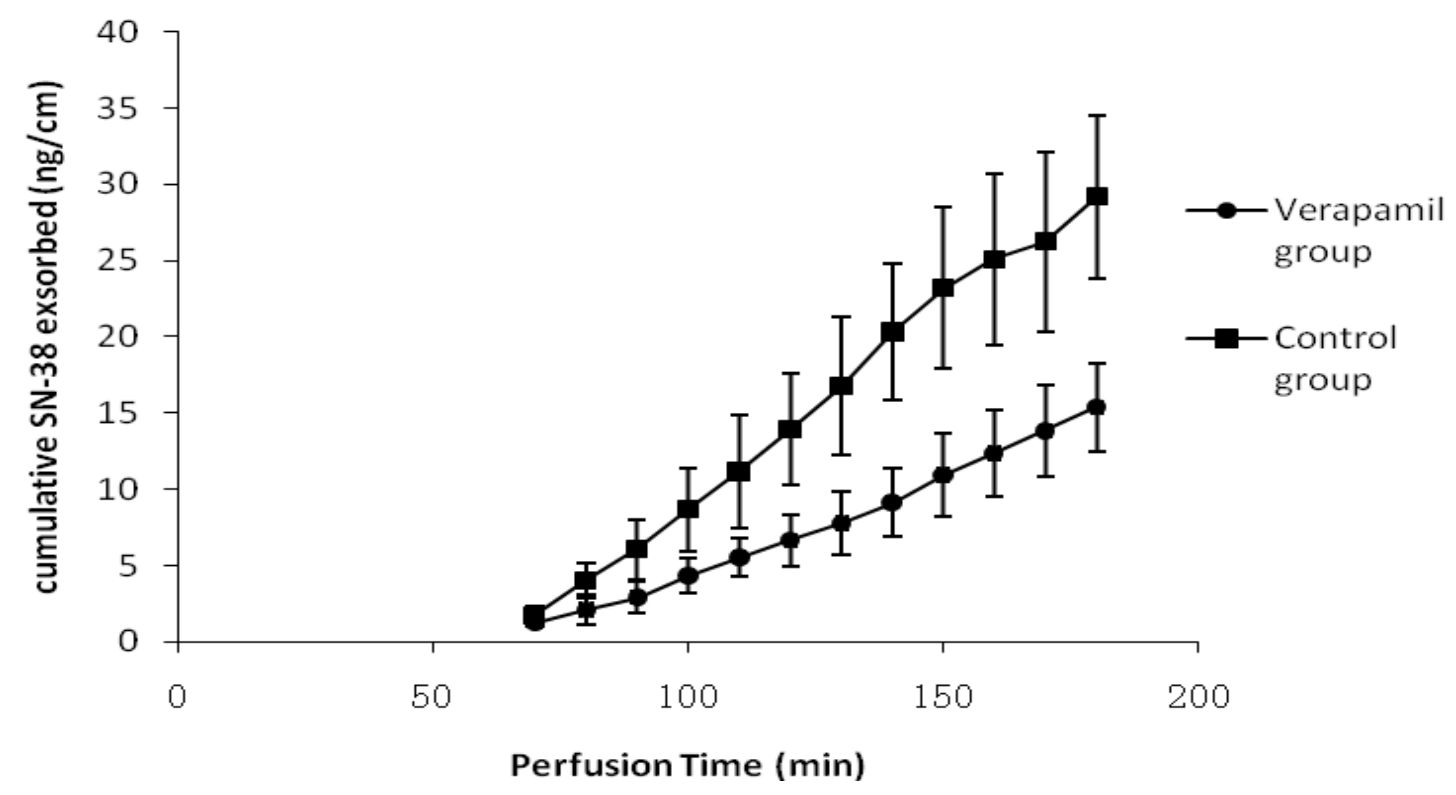

Figure 5. The cumulative exsorbed SN-38 that resulted from perfusion of irinotecan with or without the p-gp inhibitor verapamil $(P=0.016)$. Error bars represent the standard deviation of $n=6$. 
Intestinal permeability for a variety of drugs obtained from in situ single pass intestinal perfusion experiments have shown excellent correlation with human perfusion studies $(11,22)$. Also, in situ intestinal permeability of nine drugs were well correlated with corresponding human results in terms of whether these drugs being actively absorbed or they are subject to simple passive diffusion (12). In addition, permeability parameters obtained from in situ intestinal perfusion model provided a better prediction of human absorption than the cell-based assays (23).

The improved form of the intestinal perfusion technique, where the whole small intestine was used, allowed not only accurate determination of the permeability of the drug, but also gave information on the toxicity predicted due to SN-38 intestinal exposure, since the perfusion of the drug along the whole small intestines can give rise to quantifiable amounts of the active metabolite that is intestinally exsorbed.

The fact that excretion (exsorption) can give rise to appreciable concentration of $\mathrm{SN}-38$ in the gut's lumen was previously demonstrated using the in situ intestinal perfusion technique following intravenous administration (5). And since the intestinal exposure to $\mathrm{SN}-38$ in the gut was associated with the development of severe toxicity manifested as drug induced diarrhea [3, 4], we suggested that quantifying the level of SN-38 that is exsorbed following intestinal perfusion of irinotecan shall be a good indicator for predicting irinotecan induced intestinal toxicity that results from drug administration.

Although drug-drug interactions of irinotecan and $\mathrm{SN}-38$ with verapamil have already been described in previous works using $\mathrm{Caco} 2$ cells model and in vivo in rats (8), applying the improved in situ model provided two main additions, the first, is that it allowed the determination of the intestinal permeability of the parent drug, which is, to our best knowledge, the first published work providing the $\mathrm{P}_{\text {eff }}$ of irinotecan, the second, is that it allowed the quantification of $\mathrm{SN}-38$ exposure at the site where toxicity takes place.

\section{CONCLUSION}

Our HPLC-UV analytical method for the determination of the prodrug irinotecan and its active metabolite $\mathrm{SN}-38$ was validated and found to be simple, specific, accurate, and precise. And used to apply the improved rat's in situ single pass intestinal perfusion (SPIP) technique to assess the permeability, and intestinal toxicity of irinotecan predicted to result from exsorbed SN-38. The effects of P-gp inhibitor verapamil were also successfully evaluated and found to increase the intestinal permeability and potentially decrease the intestinal toxicity encountered upon the administration of irinotecan. P-gp inhibitors interaction with irinotecan necessitates future efforts to evaluate their potential to improve the oral bioavailability and ameliorate intestinal toxicity of irinotecan. This method provides a useful tool for predicting oral absorption of irinotecan and the accompanying exsorbed SN-38.

This method will be applied in our lab to assess the effect of different compounds for their potential to improve the intestinal permeability and decrease the toxicity of irinotecan for the purpose of evaluating novel oral formulations of irinotecan based on p-gp inhibition.

\section{ACKNOWLEDGMENTS}

This work was supported by the National Natural Science Foundation of China, project No. 30873171. One of the authors, Rabba AK, is grateful to the China Scholarship Counsel (CSC) for providing his $\mathrm{PhD}$ scholarship.

\section{REFERENCES}

1. Mathijssen RH, van Alphen RJ, Verweij J, Loos WJ, Nooter K, Stoter G, Sparreboom A. Clinical pharmacokinetics and metabolism of irinotecan (CPT-11). Clin Cancer Res, 2001 Aug; 7(8):2182-94.

2. U. Vanhoefer, A. Harstrick, W. Achterrath, S. Cao, S. Seeber and Y.M. Rustum, Irinotecan in the treatment of colorectal cancer: Clinical overview J. Clin. Oncol, 19 (2001), p. 1501.

3. Yang, X., Hu, Z., Chan, S.Y., Chan, E., Goh, B.C., Duan,W., Zhou, S. Novel agents that potentially inhibit irinotecan-induced diarrhea. Curr. Med. Chem, 2005. 12, 1343-1358

4. Araki, E., Ishikawa, M., Iigo, M., Koide, T., Itabashi, M., Hoshi, A., Relationship between development of diarrhea and the concentration of SN-38, an active metabolite of CPT-11, in the intestine and the blood plasma of athymic mice following intraperitoneal administration of CPT-11. Jpn. J. Cancer Res, 1993, 84, 697-702.

5. Arimori K, Kuroki N, Hidaka M, Iwakiri T, Yamsaki K, Okumura M, Ono H, Takamura N, Kikuchi 
M, Nakano M. Effect of P-glycoprotein modulator, cyclosporin $\mathrm{A}$ on the gastrointestinal excretion of irinotecan and its metabolite SN-38 in rats. Pharm Res, 2003 Jun; 20(6):910-7.

6. Gelderblom, H. A., de Jonge, M. J. A., Sparreboom, A., and Verweij, J. Oral topoisomerase I inhibitors in adult patients: present and future. Invest New Drug, 1999. 17: 401-415,

7. Yamamoto, W., Verweij, J., de Bruijn, P., de Jonge, M.J., Takano, H., Nishiyama, M., Kurihara, M., Sparreboom, A., Active transepithelial transport of irinotecan (CPT-11) and its metabolites by human intestinal Caco-2 cells. Anticancer Drug, 2001. 12, 419-432.

8. Bansal T, Awasthi A, Jaggi M, Khar RK and Talegaonkar S. Effect of P-glycoprotein inhibitor, verapamil, on oral bioavailability and pharmacokinetics of irinotecan in rats. Eur J Pharm Sci 2009; 36: 580-590.

9. Bansal T, Awasthi A, Jaggi M, Khar RK, Talegaonkar S. Pre-clinical evidence for altered absorption and biliary excretion of irinotecan (CPT-11) in combination with quercetin: possible contribution of P-glycoprotein. Life Sci. 2008 Aug 15; 83(7-8):250-9.

10. Amidon GL, Sinko PJ and Fleisher D. Estimating human oral fraction dose absorbed: a correlation using rat intestinal membrane permeability for passive and carrier mediated compounds. Pharm Res, 1988; 5: 651-654.

11. Amidon GL, Lennernas H, Shah VP, and Crison JR. A theoretical basis for a biopharmaceutic drug classification: the correlation of in vitro drug product dissolution and in vivo bioavailability. Pharm Res, 1995; 12: 413-420.

12. Varma MVS, Kapoor N, Sarkar M and Panchagnula R. Simultaneous determination of digoxin and permeability markers in rat in situ intestinal perfusion samples by RP-HPLC. J Chromatogr B, 2004; 813: 347-352.

13. Varma MVS, Panchagnula R. Prediction of In Vivo Intestinal absorption enhancement on P-glycoprotein inhibition, from rat in situ permeability. J Pharm Sci, 2005; 94: 1694-1704.

14. Li M, Si L, Pan H, Rabba AK, Yan F, Qiu J, Li G. Excipients enhance intestinal absorption of ganciclovir by P-gp inhibition: assessed in vitro by everted gut sac and in situ by improved intestinal perfusion. Int J Pharm, 2011 Jan 17;403(1-2):37-45.

15. D'Esposito F, Tattam BN, Ramzan I and Murray M. A liquid chromatography/ electrospray ionization mass spectrometry (LC-MS/MS) assay for the determination of irinotecan (CPT-11) and its two major metabolites in human liver microsomal incubations and human plasma samples. $J$ Chromatogr B 2008; 875: 522-530.

16. Bardin S, Guo W, Johnson JL, Khan S, Ahmad A, Duggan JX, Ayoub J and Ahmad I. Liquid chromatographic-tandem mass spectrometric assay for the simultaneous quantifi cation of Camptosar and its metabolite $\mathrm{SN}-38$ in mouse plasma and tissues. J Chromatogr A, 2005; 1073: 249-255.

17. Sai K, Kaniwa N, Ozawa S and Sawada J. An analytical method for irinotecan (CPT- 11) and its metabolites using a high-performance liquid chromatography: parallel detection with fl uorescence and mass spectrometry. Biomed Chromatogr, 2002; 16: 209-218.

18. Bansal T, Awasthi A, Jaggi M, Khar RK and Talegaonkar S. Development and validation of reversed phase liquid chromatographic method utilizing ultraviolet detection for quantifi cation of irinotecan (CPT-11) and its active metabolite, $\mathrm{SN}-38$, in rat plasma and bile samples: application to pharmacokinetic studies. Talanta 2008a; 76: 1015-1021.

19. Venkatesh G, Ramanathan S, Nair NK, Mansor SM, Sattar MA, Khan MA, Navaratnam V. Permeability of atenolol and propranolol in the presence of dimethyl sulfoxide in rat single-pass intestinal perfusion assay with liquid chromatography/UV detection. Biomed Chromatogr, 2007 (5):484-90.

20. Zakeri-Milani P, Barzegar-Jalali M, Tajerzadeh H, Azarmi Y, Valizadeh H. Simultaneous determination of naproxen, ketoprofen and phenol red in samples from rat intestinal permeability: HPLC method development and validation. J Pharm and Biom Anal, 39 (2005) 624-630.

21. Topic Q2B: Validation of analytical procedures: Methodology, in: International Conference on Harmonisation (ICH), 1996.

22. Zakeri-Milani $\mathrm{P}$, Valizadeh $\mathrm{H}$, Tajerzadeh $\mathrm{H}$, Azarmi Y, Islambolchilar Z, Barzegar S, Barzegar-Jalali M. Predicting human intestinal permeability using single-pass intestinal perfusion in rat. J Pharm Pharm Sci. 2007; 10(3):368-79.

23. Salphati L, Childers K, Pan L, Tsutsui K, Takahashi L. Evaluation of a single-pass intestinal-perfusion method in rat for the prediction of absorption in man. J Pharm Pharmacol, 2001, Jul; 53(7):1007-13. 